Service social

\title{
La famille et l'instruction, la pauvreté et l'hygiène publique sous l'oeil de l'histoire
}

\section{Robert Bastien et Jean-François Laé}

Volume 57, numéro 2, 2011

URI : https://id.erudit.org/iderudit/1006299ar

DOI : https://doi.org/10.7202/1006299ar

Aller au sommaire du numéro

\section{Éditeur(s)}

École de service social de l’Université Laval

ISSN

1708-1734 (numérique)

Découvrir la revue

Citer cet article

Bastien, R. \& Laé, J.-F. (2011). La famille et l'instruction, la pauvreté et l'hygiène publique sous l'oeil de l'histoire. Service social, 57(2), 113-128.

https://doi.org/10.7202/1006299ar
Résumé de l'article

L'hygiène publique et l'instruction publique sont des entrées par lesquelles on peut voir la place qu'occupent la famille, et particulièrement les familles pauvres, dans la société du XIX ${ }^{\mathrm{e}}$ et du XX $\mathrm{XX}^{\mathrm{e}}$ siècle. L'exploitation d'un fonds d'archives de la Commission scolaire de Montréal (CSDM), matière première de cet article, ouvre plusieurs voies. Ce fonds met en lumière les dispositifs d'inspection de tous les membres de l'école, les problèmes qui menacent la santé et la réussite scolaire des jeunes élèves, ainsi que les dispositifs de gestion et de contrôle que l'école et l'hygiénisme mettent en place. On y voit se déployer la " propagande hygiéniste » sur plusieurs niveaux, dans les manuels scolaires, mais aussi par une lutte acharnée pour contrer l'ignorance des familles. Manifestement, la famille est perçue comme un frein, les mères pauvres en particulier, tant elles bloquent la chaîne des opérations de l'hygiénisme. Le rôle de l'État libéral est examiné, sinon critiqué pour son laisser-aller. La science, la technique, le clergé et la morale forment un tout unifié sous une même volonté d'industrialisation : produire des hommes et des femmes en bonne santé, dociles et aptes à répondre aux exigences du monde du travail. Pour l'étude du rôle des familles dans le dessein de l'éducation et de la santé des enfants d'aujourd'hui, le détour par le passé s'impose pour connaître cette histoire qui continue d'agir. 


\title{
La famille et l'instruction, la pauvreté et l'hygiène publique sous l'œil de l'histoire*
}

\author{
Robert Bastien \\ Jean-François Laé
}

\section{RÉSUMÉ}

L'hygiène publique et l'instruction publique sont des entrées par lesquelles on peut voir la place qu'occupent la famille, et particulièrement les familles pauvres, dans la société du XIX et du $X X^{e}$ siècle. L'exploitation d'un fonds d'archives de la Commission scolaire de Montréal $\left(\mathrm{CSDM}^{1}\right)$, matière première de cet article, ouvre plusieurs voies. Ce fonds met en lumière les dispositifs d'inspection de tous les membres de l'école, les problèmes qui menacent la santé et la réussite scolaire des jeunes élèves, ainsi que les dispositifs de gestion et de contrôle que l'école et l'hygiénisme mettent en place. On y voit se déployer la « propagande hygiéniste » sur plusieurs niveaux, dans les manuels scolaires, mais aussi par une lutte acharnée pour contrer l'ignorance des familles. Manifestement, la famille est perçue comme un frein, les mères pauvres en particulier, tant elles bloquent la chaîne des opérations de l'hygiénisme. Le rôle de l'État libéral est examiné, sinon critiqué pour son laisser-aller. La science, la technique, le clergé et la morale forment un tout unifié sous une même volonté d'industrialisation : produire des hommes et des femmes en bonne santé, dociles et aptes à répondre aux exigences du monde du travail. Pour l'étude du rôle des familles dans le dessein de l'éducation et de la santé des enfants d'aujourd'hui, le détour par le passé s'impose pour connaître cette histoire qui continue d'agir.

Mots clés : Pauvreté; famille; santé; prévention; état libéral, hygiénisme; santé publique.

\begin{abstract}
The analysis of 19th and 20th century public school archives has much to teach us about the place that family - especially the poor family - has had within society. These records reveal much about the administration of public hygiene and education. They uncover specific methods in the health inspection and the management of students. They illuminate the healthcare problems of the day and the administration's attempts to mitigate or counter risks to health and scholarly success. " Hygiene propaganda » is exposed. Families - rich or poor, and certainly those burdened by social inequality - are viewed with circumspection while the role of the liberal state is questioned, if not criticised. If one wishes to study social inequalities in health and education, one would do well to study these historical records and understand their continuing impact in today's society.
\end{abstract}

Key words: Poverty, family, social and health inequalities, liberal state, public health.

* Nous tenons à remercier les professionnels et techniciens du secteur des Archives de la Commission scolaire de Montréal (CSDM) et plus particulièrement mesdames Marie-Josée Blais et Annie Plamondon, techniciennes en documentation. Enfin, nous voudrions aussi souligner la contribution de Geneviève Audet, chercheuse postdoctorale, Chaire de recherche du Canada sur l'Éducation et les rapports ethniques, Université de Montréal, Boursière FQRSC. Nos remerciements s'adressent aussi à Lucie Roy-Mustillo pour la révision de l'article.

1. Avant 1998, la CSDM portait le nom de Commission des écoles catholiques de Montréal (CECM). 


\section{Contexte}

«La médecine n'a pas seulement pour objet d'étudier et de guérir les maladies, elle a des rapports intimes avec l'organisation sociale; quelquefois, elle aide le législateur dans la concertation des lois, souvent elle éclaire le magistrat dans leur application et toujours elle veille, avec l'administration au maintien de la santé publique. Ainsi appliquée aux besoins de la société, cette partie de nos connaissances constitue l'hygiène publique et la médecine légale ${ }^{2}$. »

Dès 1829, cette première déclaration inaugurale de la Revue des Annales inscrit la médecine dans le droit et la santé publique et dans les politiques gestionnaires. Elle ne laisse aucun doute sur la volonté de gérer les populations en catégories saisissables, comme un objet politique, une biopolitique selon Michel Foucault (1975), mariant des ordres qui étaient auparavant dispersés. L'observation médicale de l'enfance scolarisée s'est institutionnalisée par ce projet de longue haleine, comme un domaine particulier de la protection sociale, contre tous les dangers qui menacent l'organisation sociale du fait de la maladie.

Au début du XIX ${ }^{\mathrm{e}}$ siècle, les pauvres sont encore écartés de la société, regardés comme une tribu à la marge, tant la pauvreté est une menace pour l'État libéral. Vecteurs de contagion, inspirateurs de débauche, de perdition, ressentis comme dangereux, les pauvres sont marginalisés et lorsqu'ils seront admis au sein de la société, ils le seront sous le contrôle d'une surveillance accrue. Par l'effet du temps, les dénominations péjoratives du pauvre instruites par les hautes classes se sont légèrement adoucies. Mais quel que soit l'angle adopté, la condamnation du pauvre à sa condition semble inaliénable, perpétuelle et durable (Castel, 1995). Bien que le vocabulaire sur le pauvre se soit modifié, on présume, toujours et encore, que certaines familles sont inaptes et qu'il faut les assister dans les tâches d'éducation, de prévention et de soins. Pauvres et familles pauvres, aujourd'hui, restent logés dans des catégories sociales de vulnérabilité, de risques et d'incompétence (Castel, 2007; Miron, 2004; René, Laurin et Dallaire, 2009; Perreault, 2008). La nouvelle terminologie, plus furtive et surtout plus savante et technique qu'auparavant, atténue les courbes d'un problème social en mettant toujours en évidence l'inertie de l'État, problème déjà relevé par des médecins hygiénistes à la fin du XIX ${ }^{e}$ siècle, et la responsabilité du pauvre face à sa propre condition (Beck, 2003). Avec le nouveau vocabulaire du pauvre, toujours localisé dans le registre du blâme (Douglas, 1992), les dispositifs de surveillance et de redressement ne se sont pas évaporés, ils se sont sophistiqués, comme l'aura révélé Foucault (1975) dans Surveiller et punir.

Les familles - indépendamment de leur condition, de leur origine et de leur statut dans les idées politiques contemporaines en matière d'éducation et de santé - sont dorénavant sollicitées à des fins de participation sociale (Moore et Lasky, 2001; Baillergeau, Thirot et Estany, 2010) alors qu'elles étaient auparavant exclues. Voilà la résultante de novateurs et de groupes militants (Donzelot, 2005) dont les luttes remontent au début du XIX ${ }^{\mathrm{e}}$ siècle. Pourtant, dans la pratique et dans le temps présent, les familles accablées par le poids des inégalités sociales, économiques et de santé ont-elles les moyens et le choix de participer à la vie

2. Extrait de la présentation de la revue Annales d'hygiène publique et de médecine légale, 1829, p. 5. 
démocratique? Et les politiques publiques et sociales ont-elles les moyens de leurs ambitions? Dans la première partie du texte, nous passerons en revue différentes déclinaisons du pauvre en tentant de situer les moments de l'histoire, les origines, les motifs et les usages de ces dénominations.

Dans un deuxième temps, notre attention se portera sur un fonds d'archives de la CSDM qui remonte au dernier quart $\mathrm{du} \mathrm{XIX}^{\mathrm{e}}$ siècle, moment où l'hygiène publique s'est instaurée à la Commission des écoles catholiques de Montréal (CECM). L'exploitation de ce fonds permet de situer la place qu'occupe l'hygiène à l'école et d'en saisir l'évolution. On y retrouve, entre autres, une importante correspondance administrative entre les autorités sanitaires, le clergé, la municipalité et la direction de la CECM, des outils d'enquête de même que des résultats d'enquêtes sur la santé des élèves et des enseignants. Ce fonds révèle aussi la manière dont l'hygiène est conçue comme matière enseignée et à enseigner ${ }^{3}$, mais plus encore comme outil de propagande ${ }^{4}$ (sic), avant que le terme ne devienne péjoratif. Subtilement, l'exploitation de ce fonds d'archives de la CECM donne à voir la place incertaine que la famille, en particulier la famille pauvre, occupe dans le dessein de l'éducation des enfants et de leur hygiène.

Aujourd'hui, les parents, quel que soit leur positionnement dans l'échelle sociale, ne sont plus formellement des cibles de la santé publique et de l'instruction. Ils sont, dans les rhétoriques sanitaires et éducatives, de vrais partenaires de la santé et de l'éducation. Cependant, dans les pratiques courantes, des embûches de toutes sortes se lèvent, trop souvent, freinant leur implication et leur participation sociale et citoyenne. Des différences ethnoculturelles liées à la maîtrise de la langue, française ou anglaise, limitent leur implication à titre de véritables partenaires (Bastien et Turcotte, 2010). Les structures de concertation, parfois, sont instituées sur des bases qui placent les parents dans des cas d'exigences avant d'espérer pouvoir participer (Bastien et Turcotte, 2010b). Enfin, la pauvreté représente toujours le principal dispositif de restriction de choix et de droits à la participation citoyenne (Bourdieu, 1993; Fleurbaey et coll., 1998; Warin, 2010; Baillergeau et Bellot, 2007; Moles, 1993).

\section{REPÈRES CONCEPTUELS}

Cet article a pour point de départ une démarche de recherche ethnographique portant sur l'implantation d'une approche en promotion de la santé en milieu scolaire dont l'un des objectifs est de susciter, dans une optique partenariale et participative, l'implication des communautés et des parents ${ }^{5}$. Émanant de nos observations et entretiens sur le terrain, notre attention s'est peu à peu orientée vers des archives de la CSDM en vue d'apporter des éléments de réponse concernant les obstacles contemporains qui freinent ou limitent la participation citoyenne des parents dans les domaines de la promotion de la santé en milieu scolaire québécois. En

3. L'inscription obligatoire de l'enseignement de l'hygiène à l'école remonte à 1892. Source : Conseil d'hygiène de la province du Québec, 28 juillet 1892. Fonds CECM.

4. À titre d'exemple, prenons le «Five o'clock tea, a sketch on milk » du Dr Adrien Plouffe qui fait partie d'un module intitulé « Sketches for Health Propaganda ». Fonds CECM, autour de 1933.

5. Devis de recherche - R. Bastien, A. Bilodeau, Y. Couturier, C. Clavier, M. Deschesne, S. Gagnon, M. Potvin et P. Warin, «Étude de la mise en œuvre de l'approche "École en santé" dans la région de Montréal : focale sur les pratiques des agents pivots », Organismes financeurs : Centre Léa-Roback, MSSS, 2009-2010. 
explorant ce fonds d'archives, notre intention était de soumettre les intentions contemporaines de la promotion de la santé à l'épreuve de l'histoire. Or, ce fonds d'archives révèle bien plus que nous le présumions, et ce que nous présentons dans cet article n'en donne qu'un bref aperçu.

La discipline hygiéniste est ici saisie comme un élément constitutif de l'exercice d'une biopolitique, si l'on suit les enseignements de Michel Foucault en matière de discipline et d'institution. L'espace disciplinaire, selon Michel Foucault (1975), est un dispositif qui associe les techniques d'intervention, les langages spécialisés et les examens individuels qui, ensemble, localisent l'exercice du pouvoir. C'est ce cadre analytique qui est ici privilégié, tant il est vrai que c'est le corps de l'enfant pauvre qui sera mis en exergue par la médecine scolaire.

\section{IMAGES DU PAUVRE}

Hier et aujourd'hui, comment nomme-t-on les personnes pauvres et quels qualificatifs s'ajoutent à leur condition? Qui se charge des dénominations, dans quel dessein et pour quels usages? Divers auteurs dont Pierrard (2005), Corbin (2008), Castel (1995) et Murard et Zylberman $(1978 ; 1996)$ relèvent l'existence de diverses dénominations pour désigner les pauvres dans l'histoire. On parle des nouveaux barbares et, dans le même registre, de sauvages pour qualifier les hordes d'hommes, de femmes et d'enfants venues des campagnes à la recherche de travail en usine à la fin du XIX ${ }^{\mathrm{e}}$ siècle. Castel (1995, p. 141) relève le qualificatif d'origine latine de «caïmands » qui décrit « ceux qui quémandent sans justification, les vagabonds en particulier ». On ira jusqu'à l'homme-fumier (Corbin, 2008, p. 213) et à la végétation immonde, sans négliger la vile multitude et la voyoucratie, pour qualifier les bas peuples. Ce vocabulaire, forgé au $\mathrm{XVIII}^{\mathrm{e}}$ et au XIX ${ }^{\mathrm{e}}$ siècle, ira toujours en se sophistiquant au gré du progrès des sciences et des techniques d'enquête et de surveillance.

Ainsi, plus on pénètre la vie privée des indigents, plus cette matière inspire de nouvelles appellations. Les « porte-voix » officiels $^{6}$ de la bourgeoisie libérale voient dans la pauvreté une menace constante qu'il faut à tout prix domestiquer, sinon éradiquer. L'Église pourra corroborer les jugements des classes supérieures à l'égard de l'indigence en créant, par exemple, des distinctions de classe morale (bonne et mauvaise) à l'intérieur de la pauvreté : « Soyez de bons pauvres et vous verrez la charité des riches couler à pleins bords sur toutes les misères ${ }^{7}$. »

L'hygiénisme - spécialité naissante dans la discipline médicale - est traversé par des réserves, des contradictions et des désaccords eu égard aux pauvres. Comme c'est aussi le cas du clergé, l'hygiène publique apparaît plus distante à l'égard d'une condamnation expresse du pauvre à sa condition et à son malheur. Sans doute présume-t-on, dans le cas particulier de la médecine et de l'hygiène publique, qu'elle ne peut réprouver, comme l'a déjà démontré

6. Adolphe Thiers (1797-1877), avocat et journaliste, Émile Buret, auteur d'une enquête sur la Misère des classes laborieuses en France et en Angleterre (1840) et H.-A. Frégier, chef de bureau à la préfecture de la Seine et auteur de l'ouvrage Des classes dangereuses de la population dans les grandes villes, et des moyens de les rendre meilleures, Éd. J. B. Baillière, 1840, 2 vol, t. 1.

7. Extrait du Manuel de charité du missionnaire apostolique Isidore Mullois cité dans Pierrard (2005, p. 51). 
Foucault (1975), avec les mêmes convictions ceux et celles qui forment la matière constitutive de la discipline : le corps, l'esprit et l'âme de l'homme qu'il soit pauvre ou riche.

En 1876, un ouvrage du $D^{r}$ Napias intitulé Le mal de la misère relève les écarts en termes de soins et d'espérance de vie entre les enfants nés dans la richesse et ceux nés dans la pauvreté $^{8}$. D'autres médecins, souvent ceux qui sont en contact direct avec les pauvres, iront dans le même sens en prenant le rôle de lanceurs d'alerte, aux côtés de notables et d'avantgardistes. Mais déjà à l'aube de la santé publique moderne, les progrès qu'annoncent les sciences et les techniques seront rapidement contrariés. D'une part, l'État libéral, trop soucieux de la sympathie de son électorat ${ }^{9}$, ne fait qu'émettre des lois facultatives (non obligatoires) censées garantir la sécurité, le bien-être et la santé de tous. D'autre part, l'exploitation croissante des journaliers et des manœuvres, enfants compris, est requise pour assurer le plein rendement des manufactures et des usines. L'inertie de l'État apparaît. La dépression économique, les baisses de salaire qu'induisent la concurrence, la guerre, les épidémies, dont la tuberculose, et les inégalités sociales et de santé sont d'autres facteurs de contrariété pour l'hygiène publique et, par le fait même, pour l'instruction publique.

De l'autre côté, on retrouve une croisade hygiéniste passablement plus hostile et plus rude à la condition du pauvre. II ne s'agit plus alors de viser l'inertie de l'État libéral qui participerait à la production du pauvre; le pauvre, ici, est saisi à partir d'une «politique négative des populations » qui donne à l'État le « devoir de dépeupler » ${ }^{10}$ :

II est peut-être choquant, déclarait un philanthrope anglais, quand il s'agit d'hommes créés à l'image de Dieu, mais il est vrai de dire que l'extinction des sous-emplois ajouterait à la richesse du pays ${ }^{11}$.

Comme nous venons de l'exposer, on comprend par les termes qui le désignent que le pauvre fait peur, le pauvre menace. Et cela, pour plusieurs raisons: par ses odeurs incommodantes et son mode de vie nomade, par les risques de contagion qu'il incarne et que les autorités médicales lui attribuent. Ces discours font de lui un être par défaut qui est condamné dès sa naissance à être inspecté. Aussi, l'indigent engendre la crainte parce qu'il menace de l'intérieur les classes supérieures, entre autres dans les villes et particulièrement dans celles qui naissent avec l'industrialisation. Pour l'éradiquer, il faut soustraire l'hommefumier à l'autorité des sens : la vue, l'ouïe, l'odorat et le toucher.

8. « Le premier [l'enfant né dans la richesse] connaîtra une longue vie sans heurts, alors que l'enfant né dans la misère, le manque et la pauvreté, aura une vie nécessairement plus courte. » Extrait du Mal de la misère (1876) cité dans l'ouvrage Les pauvres et leur histoire de Pierre Pierrard (2005, p. 38).

9. Rappelons la mise en forme d'une loi en 1850 qui annule toute participation des ouvriers itinérants au droit de vote en France. Ainsi, de 9600000 , le nombre d'électeurs passe à 6800000 . À Paris, dans l'ancien XIle arrondissement, le nombre d'électeurs chute à 110000 alors qu'il était de 353000 avant l'application de la loi (Pierre Pierrard, 2005, p. 40).

10. L. Murard et P. Zylberman, « Hygienic preservative against various maladies », dans Lion Murard et Patrick Zylberman (dir.), L'haleine des faubourgs : ville, habitat et santé au XIX siècle, revue Recherches, $\mathrm{n}^{\circ} 29,1978$, p. 7-22.

11. S. Barnett (1888) cité par G. S. Jones, Outcast London - A study in the relationship between classes in Victorian Society, Oxford, Oxford University Press, 1971. Cité dans L. Murard et P. Zylberman (1978, p. 14). 
Donzelot (2005) identifie un moment charnière de l'histoire où la mise au rancart du pauvre se transforme en même temps que se modifient les dénominations abjectes. Être irréparable à plusieurs égards et condamné à son sort, le pauvre devient subitement utile par sa force de travail que la bourgeoisie réclame pour ses menées extérieures (Donzelot, 2005, p. 161). Sorte de chair à canon, le pauvre qui auparavant était contraint à freiner sa reproduction sous prétexte qu'il perpétuait sa race immonde, voilà que pour les efforts de guerre, pour cause de dénatalité et pour d'autres motifs idéologiques, politiques et économiques, il doit redevenir fertile et se perpétuer pour le bien-être de la Nation et le profit d'autrui. Augmenter la productivité et la rentabilité de la vile multitude suppose d'aller plus loin que l'assistance de l'État et de l'Église. II faut dorénavant éduquer cette classe malpropre et dangereuse ${ }^{12}$ (Faure, 1978), l'instruire et lui inoculer des savoirs capables de la rendre docile et adaptée aux exigences du travail à la manufacture. Autrement dit, faire du pauvre un citoyen respectueux de soi comme des autres et sensible aux méfaits des mauvaises mœurs. En matière d'éducation, l'opération ne fait pas l'unanimité, car on présume que l'instruction publique de tous et de toutes pourrait chambarder l'ordre social.

L'instruction est un moyen de perfectionnement et de bonheur pour l'homme, comme elle peut être pour ce dernier un instrument de corruption et de ruine; c'est une lumière qui éclaire ou qui brûle, selon qu'elle est bien ou mal dirigée. Tel est, malgré tous les paralogismes, l'effet certain de l'instruction considérée dans ses rapports, soit avec le riche, soit avec le pauvre. Cependant, l'instruction du premier étant plus complète que celle du second, et les loisirs dont il jouit lui permettant également d'étendre ses lumières et d'épurer son cœur, on ne saurait établir de comparaison, sous ces deux rapports, entre lui et le pauvre, qui, par sa condition, est destiné à un labeur perpétuel et ne peut, dès lors, espérer acquérir que des notions imparfaites en toutes choses, même en morale. L'acquis de l'esprit chez le pauvre, pour cela qu'il est superficiel et incomplet, doit donc être soumis, de la part de ceux qui gouvernent, à une surveillance perpétuelle ${ }^{13}$.

Comme il appert dans cette déclaration, on suggère que le pauvre est inapte à apprendre et à se soumettre aux exigences de l'instruction alors réservée à l'élite. Par compromis, on lui donnera donc uniquement des bases académiques élémentaires, affirmant que les enfants des pauvres tendent à répudier l'école et que, de toute façon, leur avenir se trouve dans les mines, à l'usine ou à la manufacture ${ }^{14}$. La mise en place de l'instruction publique au Québec soulève les passions. D'une part au sein du clergé, qui voit dans l'éducation le véhicule premier de la civilisation de l'homme, mais aussi au sein de la bourgeoisie canadienne qui cherche à s'approprier ce champ au profit de l'Église (Gagnon, 1996).

12. Titre d'un des chapitres (p. 79-102) du livre L'haleine des faubourgs (L. Murard et P. Zylberman, 1978).

13. H.-A. Frégier, Des classes dangereuses de la population dans les grandes villes, et des moyens de les rendre meilleures, Éd. J. B Baillière, t. 2, 1840, p. 1.

14. L'instruction publique est soumise à d'importantes pressions externes qui lui feront perdre des élèves aspirés par le monde du travail, qui embauche des enfants qui n'ont que six ou sept ans. Or, l'État libéral est amené à modifier des lois pour s'assurer de la fréquentation des écoles par les élèves. C'est ainsi que l'on abroge la loi sur les manufactures afin de hausser à 14 ans l'âge minimal des nouveaux journaliers (Gagnon, 1996, p. 126). 
Pour illustrer les idées politiques, qui nous paraissent aujourd'hui iniques, qui avaient cours à l'aube de l'instauration de l'instruction publique destinée aux classes laborieuses, citons un extrait du $D^{r}$ Jean-Baptiste Meilleur ${ }^{15}$, alors surintendant au Bas-Canada, rapporté par Gagnon (1996, p. 27) :

[Meilleur ne veut pas, par exemple,] pour la masse des enfants du peuple d'une éducation savante et classique, d'une haute éducation, enfin, parce que cette espèce d'éducation serait superflue et inutile pour elle et que la société pourrait plutôt en souffrir qu'en profiter sous le rapport moral et matériel. Cette espèce d'éducation étant un luxe, et généralement du ressort des parents aisés, ne doit être qu'à leurs enfants, à ceux de l'élite de la société et autant que possible, à tous les autres enfants ayant une aptitude spéciale, afin de former les uns et les autres pour les professions et pour les plus hautes positions de la société.

\section{HYGIÈnE PUBLIQUE ET INSTRUCTION PUBLIQUE : UNE INCURSION DANS DES ARCHIVES}

Au Québec, à la fin du $X I X^{e}$ siècle et au début du $X X^{e}$ siècle, trois institutions intimement liées les unes aux autres participent au développement de l'instruction publique : le clergé, l'État et l'hygiène publique. À l'école, l'hygiène publique se décline en quatre principaux versants. Premièrement, il s'agit de réaliser des examens répétés sur le corps, l'âme et l'esprit de tous les membres de l'école : concierges, enseignants, élèves, etc. Deuxièmement, pour s'assurer de l'efficacité sociale de l'entreprise, en l'occurrence l'hygiène publique, il faut garantir une dissémination soutenue et massive des règles d'hygiène auprès de tous les membres de l'école, mais aussi, par la voie des médias écrits et de la radio, dans la population entière. Troisièmement, pour créer une forte cohésion interinstitutionnelle, l'hygiène publique doit se conformer aux diktats de l'Église, plus encore, s'en inspirer très fortement quant à la façon dont elle entend mettre en scène son système « d'inoculation » de connaissances ${ }^{16}$. D'ailleurs, elle ne peut échapper à l'emprise du clergé qui sanctionne tous les contenus pédagogiques et sanitaires sous toutes leurs formes, qu'il s'agisse d'écrits, d'images, de films et d'émissions radiophoniques (Paquin, 1960). Enfin, par la mise en place des mesures d'hygiène à l'école, on s'assure au présent de produire des élèves dociles, assidus et respectueux en plus de préparer,

15. Il importe sans doute de savoir que l'instruction publique s'est développée de manière séquentielle rythmée par des avancées et des reculs de 1838 jusqu'à la fin de la première moitié du XIX $\mathrm{X}^{\mathrm{e}}$ siècle. Hors de tout doute, le surintendant Meilleur provient des classes supérieures parce qu'il a bénéficié, par l'entremise de la position sociale de sa famille, d'une éducation savante. Néanmoins, il faut rappeler que l'éducation dans le Bas-Canada est dans un état lamentable dans les années 1830. II est donc impensable, pour l'époque et pour un seul homme qui milite sur tous les fronts en matière d'éducation, d'instaurer, de but en blanc, une instruction démocratique. Obligatoirement, l'institution, telle que nous la connaissons aujourd'hui, se constituera par étapes successives.

16. Voici quelques exemples de la place que le clergé occupe dans le dessein de l'hygiène publique à la fin du $X I X{ }^{e}$ siècle dans les manuels scolaires. En 1887, dans le Petit Résumé du cours d'hygiène du couvent de Sillery, l'un des sept préceptes généraux de l'hygiène se fonde sur la foi, il s'agit du septième « De la religion comme moyen d'hygiène ». On note l'existence, dès 1890, de deux ouvrages approuvés par le Comité catholique du Conseil de l'instruction publique, soit Le traité élémentaire d'hygiène privée qui se destine «à toute personne d'une préparation scientifique moyenne » et Le catéchisme d'hygiène privée qui est destiné « aux enfants pour des exercices de lecture et de mémoire ». On relève aussi, dans l'Abrégé d'hygiène du Dr Palardy de 1927, la mention suivante dans l'avant-propos: «La forme d'un bref questionnaire, d'une sorte de catéchisme de l'hygiène est apparue comme remplissant mieux que tout autre, ces conditions. » Source : Fonds d'archives de la CSDM. 
pour demain, une masse laborieuse ou encore des soldats de bonne qualité. L'industrialisation et les deux grandes guerres ont des effets structurants sur la place de l'hygiène à l'école.

\section{Rituels D'EXAMENS ET " INOCULATION " DE CONNAISSANCES AUX MASSES PAUVRES}

Cette partie de l'article pose un regard attentif sur deux versants de l'hygiène publique à l'école: (1) les dispositifs d'inspection de l'ensemble des membres de l'école, dont les élèves en particulier, et (2) la dissémination de savoirs hygiénistes à l'école et dans la société. Pour que le quadrillage disciplinaire ${ }^{17}$ produise des résultats, certaines conditions doivent être réunies. En voici six : (a) l'inspection répétée des corps et de l'esprit de tous les membres de l'école; (b) l'usage de techniques issues de la science; (c) la séparation des élèves sains des élèves contagieux, défectueux (sic), débiles (sic) et arriérés (sic); (d) la remise sur pied, lorsque possible, des élèves malades; (e) l'émission de certificats médicaux témoignant de la bonne santé des sujets inspectés et enfin, (f) l'implication des familles, et des mères en particulier qui sont censées assurer le relais entre le travail des médecins hygiénistes et l'école.

Pour que l'hygiène puisse porter ses fruits, le rôle des mères de famille est capital. Les connaissances hygiénistes dispensées à l'école doivent être reprises à la maison par les mères. Or, rapidement les médecins hygiénistes doutent des compétences des mères. Sur ce dernier point, on sait que les « basses classes » risquent de compromettre la chaîne des opérations. Les inégalités sociales qui accablent les familles pauvres liées à la piètre éducation des mères devront être comblées promptement par l'intermédiaire de la « propagande hygiéniste ».

De 1929 à $1930^{18}, 135000$ enfants scolarisés de Montréal ${ }^{19}$ sont passés à la loupe ${ }^{20}$. C'est l'heure des grands examens. Parce que le taux de mortalité des 5-14 ans est fort élevé (4 \% en 1928 dans cette tranche d'âge), parce que l'enfance indigente transporte les maladies de la maison vers les établissements scolaires, il est temps de monter une digue contre les assauts de diphtérie, de dermatoses, de gale et de teigne. Parce que les classes pauvres n'ont « ni l'intelligence ni le temps requis» pour combattre les maladies contagieuses et les « défectuosités » de leur progéniture, les infirmières visiteuses et les médecins hygiénistes de la CECM œuvrent à inscrire sur des tableaux statistiques toutes les anomalies que la science et les techniques permettent de révéler : de la dénutrition aux amygdales, du système nerveux aux maladies de la peau, du système génito-urinaire aux oreilles et aux nez, etc. À l'image du

17. Le quadrillage disciplinaire est un concept employé par Foucault, entre autres dans Surveiller et punir, pour décrire l'exercice du pouvoir des disciplines sur les hommes. C'est dans le « monde » hospitalier et carcéral que ce type de quadrillage est le plus facilement observable parce que l'humain y est soustrait au monde extérieur et isolé de lui, ce qui permet de réaliser des examens répétés sur les sujets. Ainsi les connaissances générées nourrissent et légitiment la discipline en même temps qu'elles servent à catégoriser le sujet dans les termes de la discipline.

18. Ce n'est pas la première fois qu'on se propose de faire l'examen de la population scolaire à Montréal : dix ans plus tôt, en 1919, le Service de santé de la Cité de Montréal prenait l'initiative d'une grande inspection. L'intention était toutefois restée au stade d'une simple programmation, le projet déjà bien élaboré resta lettre morte. Source : CSDM.

19. En 1931, ils seront 138 000, l'année suivante, 149 000, puis en 1934, 151 000. Source : CSDM.

20. 75 gardes-malades visiteuses, 20 médecins inspecteurs, 4 inspecteurs dentaires vont parcourir 277 écoles catholiques et protestantes pour recenser les maladies les plus flagrantes. Source : CSDM. 
service militaire, l'inspection des corps, comme des lieux physiques ${ }^{21}$, s'impose à la fois pour agir sur les maladies mais aussi sur les familles des petits malades, par des avis écrits à cellesci, par des visites à domicile pour éduquer les parents. 1929, c'est l'heure de l'exécution du dispositif (voir tableau 1). C'est le moment où on libère une salle dans chaque école qu'on pourvoit du matériel nécessaire afin d'accueillir les médecins des dispensaires et des bureaux de vaccination; où on fait passer un examen médical préalable à tout le personnel exerçant dans les établissements ${ }^{22}$; où on ouvre à l'Hôpital Notre-Dame un laboratoire d'examen de la peau pour accueillir les flux des élèves malades.

Puisque la dénutrition est d'emblée évidente, on profite de ce grand mouvement pour distribuer des bouteilles de lait d'un demiard « aux enfants pauvres fréquentant les écoles de la CECM ». Ainsi, 9332 enfants recevront à quatre reprises cet aliment, non sans qu'on observe la prise de poids au fil des semaines. C'est la goutte de lait qui doit faire ses preuves. La balancetoise est installée dans chaque salle scolaire.

On pèse, on ausculte et on mesure. Pour prolonger cette action, on distribue 5000 ouvrages gratuitement à tous les membres du personnel des écoles: le manuel du $D^{r}$ Palardy et la conférence radiophonique sur l'hygiène mentale du $D^{r}$ Barbeau prennent le relais de l'enseignement régulier de l'hygiène en classe et des causeries pédagogiques sur les vertus de l'hygiène ${ }^{23}$. On envoie six institutrices, dont quatre religieuses, à Boston pour se spécialiser dans l'enseignement des « arriérés », afin qu'elles ouvrent à terme des classes spéciales sur le modèle de l'école La Jemmerais de Québec. II en va de même pour les enfants « débiles » et les enfants « infirmes ».

21. Le premier rapport écrit (en anglais) du Bureau d'hygiène de la Mairie de Montréal remonte à 1886. On fait état du délabrement des toilettes et des urinoirs de même que du vitrage de l'école et du cubage restreint de l'air dans l'école et les classes. Un second rapport, en français, daté du 2 mai 1892 fait état de deux inspections d'école. Au sujet de l'une de ces écoles, détenue par un propriétaire privé, il est fait mention que «les arrangements sanitaires sont défectueux » et que le propriétaire a été avisé des irrégularités. Source : CSDM.

22. La question de l'examen médical du personnel enseignant et de tout autre adulte en situation d'autorité, comme le révèle une correspondance administrative entre la Commission pédagogique et le Service de santé de la Cité de Montréal, est génératrice de tension. À titre illustratif, en 1931, le Service de santé exprimait son désir que tous les instituteurs laïques et religieux soient astreints à un examen médical annuel gratuit. Or, lors d'une réunion tenue le 2 février 1932, la Commission pédagogique prit la décision de rendre facultatif pour son personnel laïque l'examen offert gratuitement par le Service de santé. Source : CSDM.

23. Prenons pour exemple un programme d'hygiène d'une semaine figurant dans une publication du Service provincial d'hygiène. Tous les jours de la semaine comportent des moments de diffusion de connaissances hygiéniques: le dimanche, on abordera à l'église l'hygiène générale. Le lundi, on montrera les bienfaits de l'hygiène. Le mardi, il sera question des maladies contagieuses, de la vaccination, des traitements antivarioliques. Et ainsi de suite. Enfin, lors de la dernière journée de la semaine, les enseignants en profiteront pour demander aux élèves de faire un « devoir » sur un sujet touchant à la santé. Source : CSDM. 


\section{Tableau 1 : L'inspection médicale des écoles}

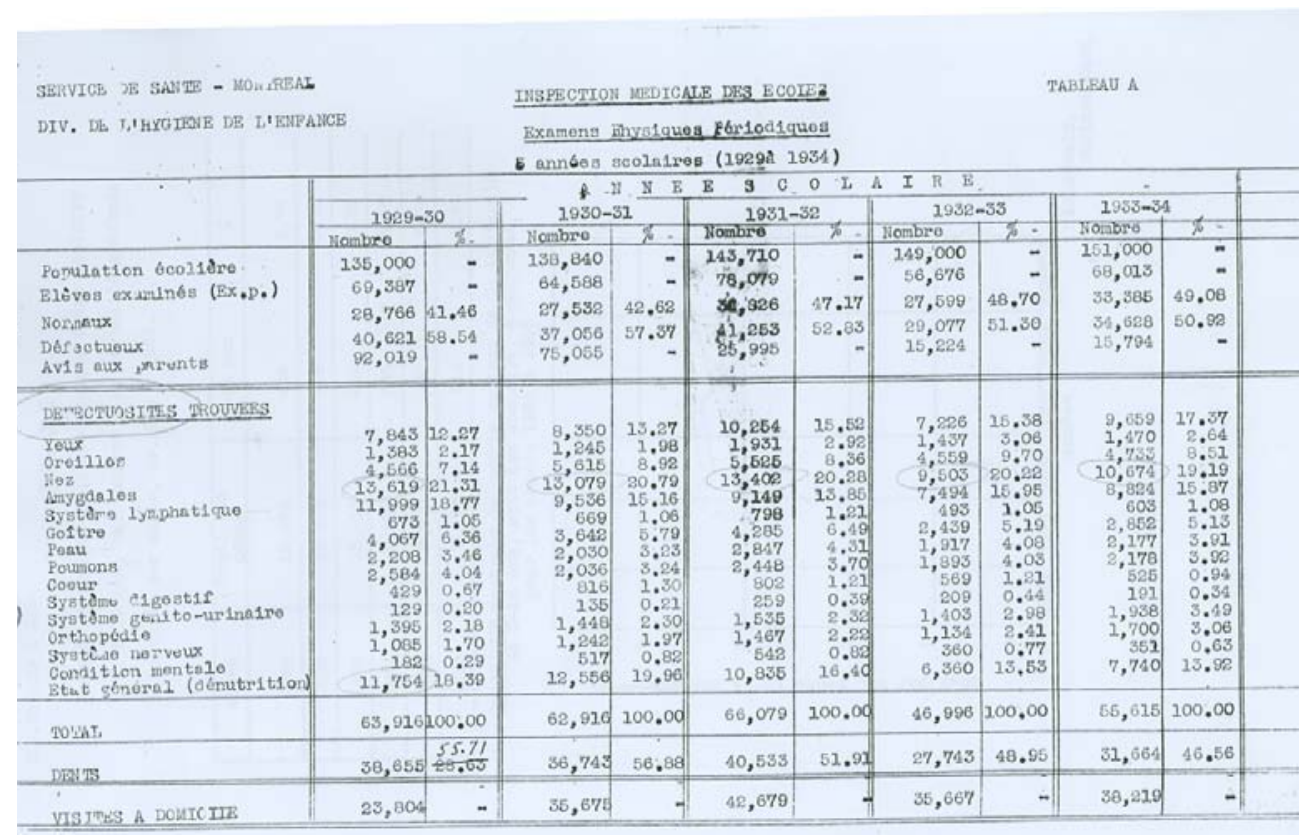

L'étonnant de ce tableau, ce sont les rubriques des défauts trouvés, qui découlent pour l'essentiel d'un regard plus hygiéniste que médical. En effet, si on regroupe la malpropreté, la pédiculose (les poux), la peau et les dents, c'est bien des conditions de vie et de l'alimentation qu'il est question. Pour chaque rubrique, c'est de 10 à $15 \%$ d'anomalies qui sont relevées, sachant qu'un seul écolier peut apparaître simultanément dans plusieurs rubriques. Sur les cinq années observées, l'inspection des écoles se satisfait des premiers résultats: tendance au recul des indicateurs négatifs qui passent globalement de $55 \%$ de défauts à $46 \%$. De même, le taux de mortalité de cette classe d'âge passe de $4 \%$ à 2,40 \%.

Cette priorité hygiéniste est remarquable à plus d'un titre. Tout d'abord, l'approche en termes de population s'oppose à celle qui individualise les problèmes du corps à partir d'un carnet de santé propre à chaque enfant. En contrepartie, ce dispositif de médecine scolaire écarte les familles de leur propre exercice sur la santé de leurs enfants. Cette éviction des familles est saisissante, soulignant un rapport social qui fait de celles-ci des familles réprouvées, déchues et blâmables ${ }^{24}$. Ainsi, les conduites sanitaires ne sauraient être exercées par « le milieu qui est

24. L'extrait qui suit est éloquent à ce sujet. « J'ai donc raison de dire qu'il faut marteler, remarteler les notions d'hygiène et de médecine préventive à l'intention des parents. (...) Les professeurs enseignent le bon langage, les enfants se surveillent en classe, mais ils retournent à la maison le soir et là, personne ne les reprend quand ils parlent mal. Ce qui est gagné à l'école est perdu à la maison! La même comédie se répète pour l'hygiène, les professeurs, les médecins et les infirmières donnent de bons conseils mais les parents ne s'en occupent pas et, trop souvent, ils s'en moquent » (Dr Plouffe, « Il faut la tuer », L'Action médicale, Montréal, juin 1933, p. 104). Source : CSDM. 
pathogène ». Ici, c'est le modèle militaire qui l'emporte. Faire un militaire, cela suppose un arrachement à toutes les valeurs et pratiques de sa famille. Faire un écolier sain, c'est révoquer la famille qui représente un danger, un empêchement, voire un péril. Le résultat attendu découle de la procédure d'inspection: plus de $58 \%$ des enfants sont «porteurs de défectuosités ». Par ordre d'importance, les problèmes d'amygdales, du système lymphatique, de la dénutrition et des yeux sont au-devant de la scène. Disons-le clairement, lorsqu'on parle d'un système lymphatique défaillant, on veut dire que le corps enflammé de l'élève découle d'une mauvaise hygiène, des piètres conditions de vie qui font de lui une personne mal armée. N'est-ce pas la saleté qui enflamme les plaies? N'est-ce pas le logement insalubre qui est accusé en même temps que ceux qui y habitent? Prenons par exemple le codage de la galle ou encore des maladies contagieuses (dans toutes les institutions militaires, industrielles, scolaires, dans la marine, les colonies), qui supposait de procéder à des analyses (symptomatologie, nosographie, pronostics, observations, dossiers cliniques) afin de faire fonctionner cette technologie de protection au nom du savoir et du pouvoir médical. II a fallu coder la galle comme danger, c'est-à-dire qu'il a fallu faire apparaître la transmission de la galle comme porteuse d'un certain nombre de dangers, comme essentiellement porteuse de périls pour ces institutions et, du coup, inventer une médecine spéciale - armée de statistiques, de médecins enquêteurs, de tableaux de bord - afin de dessiner les lieux, d'isoler, de découper les zones de danger social et de donner à la galle, en même temps, un statut de maladie, un diagnostic de pathologie.

Les archives de la CSDM montrent ainsi comment concrètement, à Montréal, à l'intérieur de la scolarité même, on fait fonctionner ce savoir sur les institutions associées à l'hygiène publique. Et c'est ainsi qu'on peut relever deux grandes opérations. L'une à l'intérieur des classes scolaires, qui consiste à bâtir des grilles de suivi, des cohortes de maladies et des points du corps de l'enfant à surveiller. L'autre, dans les lieux d'habitation de Montréal, les lieux pauvres, dégradés, les baraques en bois et auprès des familles pauvres, une lutte contre les taudis vus comme des transmetteurs des maladies de la galle. Ce double codage va connaître une longue histoire tout au long du XX $X^{\mathrm{e}}$ siècle, jusqu'en 1970 environ, et l'on peut dire que le temps fort de cette histoire sera précisément lorsque les deux codages se trouveront effectivement ajustés, lorsqu'on aura un seul et même type de discours, un seul et même type d'analyse, un seul et même corps de concepts, qui permettront de constituer la galle comme maladie et de la percevoir comme un danger social. Ce double codage est absolument nécessaire, comme une des conditions de la réussite de cette lutte, comme branche de l'hygiène publique, mais aussi comme une lutte contre la pauvreté (et par là, contre les pauvres). D'où l'intérêt essentiel, constitutif, au sens fort du terme, pour les points précis du corps; l'attention particulière aussi à toutes les formes de comportement des enfants et des familles qui sont imprévisibles et imprévoyantes à l'égard des soins à prodiguer. C'est, en quelque sorte, la prouesse d'intronisation de l'hygiénisme dans les classes pauvres. Une intronisation par des récits types, du genre : si vous avez la peau trop fragile, si vos chaussures sont mal ajustées à vos pieds, si vous ne prenez pas soin d'eux, alors les contusions de la peau provoqueront telle ou telle maladie. C'est ainsi que toutes les peaux maltraitées entrent peu à peu dans une nosographie, dans une médecine sociale adaptée aux besoins de la société. 
L'inspection médicale des écoles accuse massivement les familles (riches et pauvres) de ne pas être à la hauteur de l'éducation sanitaire de leurs enfants ${ }^{25}$ par manque d'intelligence, par ignorance et par manque de volonté. L'apathie, l'indifférence, l'imprévoyance, le manque d'attention et l'illettrisme des familles pauvres, en particulier, sont indirectement désignés. Formant un haut lieu éducatif, l'alliance de l'institution scolaire et de l'institution médicale se retourne contre le milieu malsain d'origine, les quartiers et leurs pauvres. On peut dire qu'à travers ce dispositif sanitaire, le système scolaire se met aux ordres d'une approche populationnelle, soit une démarche qui détermine dans une population donnée une légalité de la santé, avec ses désordres, une pensée négative qui désigne un reste: le pauvre et son milieu pathologique. Un seul écolier malade, et c'est toute la classe qui est infectée, même constat pour les concierges et les enseignants qu'ils soient laïques ou religieux. En exerçant une surveillance globale et totale au sein des établissements scolaires, il s'agit de faire le partage entre la bonne et la mauvaise santé, d'organiser des remparts contre les maladies contagieuses, et d'éliminer ce qui est dangereux venant du dehors. Le dehors, ici, peut être l'imprévoyance, l'ignorance, l'inintelligence des mères et le foyer familial des pauvres. L'ignorance est partout et il faut la combattre, comme l'affirme le $D^{r}$ Plouffe dans un article intitulé Il faut la tuer ${ }^{26}$.

L'inspection est une technologie statistique sur laquelle les autorités scolaires et sanitaires peuvent s'appuyer pour agir, l'État aussi. Elle renforce la puissance de l'institution scolaire, en montrant que la population des élèves a ses régularités propres : son nombre de malades, de handicapés, de malades mentaux, sa distribution de malformations et de défectuosités. Constantes et régularités statistiques forment le socle de l'action publique. La statistique montre également que cette population comporte des effets propres à son agrégation et que ces phénomènes sont réductibles à la famille. Enfin, de cette pensée en termes de population découle l'idée de propagande. Du coup, la famille pauvre (re)passe à un niveau inférieur par rapport à la population sous prétexte qu'elle est ignorante. L'art de gouverner la santé de l'élève transite maintenant par l'institution scolaire, mais aussi par le chas de l'hygiénisme qui s'adresse historiquement aux ouvriers pauvres des industries et aux pauvres de l'assistance. Avec l'hygiénisme, point besoin de théorie médicale sophistiquée, le médecin hygiéniste et l'infirmière n'ont qu'à gratter la chevelure et écarter la mâchoire de l'élève pour voir le désastre. La famille aussi a un rôle à jouer dans le quadrillage hygiéniste, mais l'on voit que celles qui sont accablées par la pauvreté sont rapidement évacuées, car on présume de leur inadéquation. Plus encore, ce sont les mères et non les pères qui sont visées par la croisade des médecins hygiénistes; les pères étant au travail, c'est la mère qui hérite de toutes les responsabilités et, du même coup, de tous les blâmes. Circonspecte voilà un peu moins de cinquante ans, qu'en est-il de la famille pauvre dans le dessein de la prévention, de la santé et de l'éducation aujourd'hui? Comme nous le disions en introduction, les qualificatifs attribués à la pauvreté et plus encore aux familles pauvres se sont adoucis. La terminologie actuelle est en

25. Dans la missive du Dr McKee (1927) de l'Hôpital de New York, il n'y a plus de distinction formelle entre famille pauvre et famille riche à l'égard des soins qu'elles ont à prodiguer à leurs enfants malades. La note est révélatrice : «This laborious and disagreeable treatment [pour contrer les teignes : ringworm] is successful when the patient's family is intelligent. » Source : CSDM.

26. L'Action médicale, Montréal, juin 1933, p. 104. Source : CSDM. 
apparence moins affligeante qu'elle l'était auparavant. Or, l'adoucissement des qualificatifs n'indique en rien la disparition de la condition sociale des pauvres, et ni non plus l'évanouissement des dispositifs de surveillance et de contrôle des enfants et des familles pauvres (Castel, 1995). Foucault (1995, p. 15) a ouvert la voie à ce type de questionnement en portant son attention sur le raffinement et la sophistication des régimes de surveillance et de punition, entre autres dans le champ pénal. C'est une punition douce dont il est question ici. Non pas la violence d'État, non pas des châtiments, non pas des sévices corporels, mais bien plus un régime punitif qui prend les corps de l'enfance pour les redresser, qui prend la famille à témoin de son incapacité, qui marche à la culpabilité et à la honte. Pénalité morale, pourrionsnous dire, après Foucault, cette façon de culpabiliser les parents, de souligner leur inaptitude, de les faire rentrer à l'école pour leur apprendre à vivre chez eux, voilà un nouveau régime punitif presque invisible qui se lève. Le punitif n'est plus la claque, mais son envers, une douce invitation à baisser la tête devant les injonctions à « bien parler la bonne langue » instruite par la discipline. Redresser la langue, c'est aussi redresser les corps, redresser la famille à cause de l'enfance.

\section{ConClusion}

Relever, consigner et s'attaquer, par la prévention, aux irrégularités physiques et psychiques qui menacent la santé des élèves et par extension, la santé de tous, telle est la mission de l'hygiène publique. Sonner des alertes partout où l'hygiène peut s'insérer et progresser, à l'usine, dans les familles, à l'école, dans les médias et dans l'État. Mais il reste des lieux où l'opération de branle-bas est plus difficile à réaliser et l'un de ces lieux est, paradoxalement, l'école elle-même. Pour que la discipline puisse espérer se constituer, l'hygiénisme doit avoir à sa disposition « des sujets qui ont à être vus » (Foucault, 1975, p. 189). C'est là la première étape de la constitution disciplinaire. À celle-ci s'en ajoute une seconde tout aussi importante, mais plus complexe à réaliser que la première. II s'agit des actions à entreprendre pour améliorer la santé du sujet lorsqu'on détecte des défectuosités. L'exemple qui suit illustre une part de la complexité qui réside dans cette double opération d'observation et d'intervention.

Par inadvertance, l'inspection de la psyché des enfants conduit à la révélation de vérités qui placent l'hygiène publique, l'hygiène mentale et l'école dans une position inconfortable. L'extrait d'une missive de deux psychiatres hygiénistes est révélateur d'un malaise au sein de deux institutions, la santé et l'éducation, qui, à l'époque, n'ont ni l'intérêt ni les moyens de se mettre en opposition.

«Dans un test de vocabulaire, nous demandions aux élèves: qu'est-ce qu'une règle? Chez les enfants d'âge mental de 8 ans ou moins, il est normal d'avoir une réponse par l'usage. (...) une fillette nous amenait comme réponse, c'est pour des barres ou c'est pour mesurer. (...) La même question chez plusieurs petits garçons lui (sic) faisait répondre : c'est pour donner la volée. Le petit définissait l'usage. (...) Or, nous savons que les enfants ne sont pas seulement punis, mais odieusement battus, au point que le bruit des coups, les cris et les gémissements (...) troublent la quiétude d'esprit et le calme dont nous avons besoin. (...) Est-ce dire qu'il faille complètement s'abstenir à l'école de punitions corporelles? Nous ne le croyons pas. (...) la punition doit être proportionnée à la gravité de la faute. (...) 
Un enfant traité avec brutalité (...) a bien des chances d'être plus tard un homme brutal $^{27}$. »

Pour finir, il faut bien mesurer la hauteur de l'enjeu, il s'agit bien d'un projet d'alliance pour inscrire la médecine dans la scolarité, d'implanter l'hygiène publique dans les politiques gestionnaires des écoliers, avec trois principaux types de techniques. Comme première technique, il y a celle qui permet de produire une population scolaire problématique semblable à l'idée de cas en médecine, à la différence que l'hygiénisme ne soigne pas, il se pose au-devant du mal par la prévoyance, par la précaution. Cette même technique légitime la production, la transformation et la manipulation de la figure de l'enfant malade dans un régime d'observation et de traitement dont se charge l'Hôpital. Comme seconde technique, consubstantielle à la première, il y a l'utilisation des systèmes de signes (la nosographie dermatologique, par exemple) dont l'efficacité permet l'invention de mots pour produire des disjonctions, de nouvelles lignes de séparation et de démarcation. Enfin, il y a les techniques qui déterminent qui doit agir sur les bénéficiaires de soins et qui doit imposer certaines finalités ou certains objectifs éducatifs à l'intérieur même du système de protection. Dans la rencontre de ces techniques, préside une question, celle de l'obligation de vérité : il faut découvrir la vérité des retransmissions, dire la vérité sur les pauvres comme agents de retransmission; il faut des professionnels qui établissent la vérité statistique, comptable, médicale, juridique des maladies infantiles. Comment peut-on dire la vérité d'un élève travaillant, mais qui ne peut plus travailler comme élève sans divulguer les lieux, la famille par exemple, sans participer à la promotion du blâme qui ne fait qu'ancrer davantage la déqualification et la disqualification des familles pauvres? Encore aujourd'hui, la solution à ce problème n'est pas trouvée. Est-ce lié au caractère public de l'investigation ou encore à la persistance des inégalités sociales et de santé? Ainsi, la nouvelle «nosopolitique » inscrit la question spécifique de la maladie des enfants pauvres dans le problème général de la santé des populations; et elle se déplace du contexte étroit des secours de charité à la forme plus générale d'une " police scolaire », avec ses contraintes et ses services.

Robert Bastien, Ph. D.

Chercheur

Direction de santé publique de Montréal

Professeur adjoint de clinique Département de médecine sociale et préventive

1'Université de Montréal

Jean-François Laé, Ph. D. Professeur

Université Paris VIII (France) Département de sociologie, UFR 04 Université Paris 8.

27. Lettre datée du 28 janvier 1939 adressée au Président du Comité national d'hygiène mentale, Section de la province de Québec. Source : CSDM. 


\section{RÉFÉRENCES BIBLIOGRAPHIQUES}

Baillergeau, É. et C. Bellot (dir.) (2007). Les transformations de l'intervention sociale. Entre innovation et gestion des nouvelles vulnérabilités?, Québec, PUQ.

Baillergeau, E., M. Thirot et M.-L. Estany (2010). « Au contact des invisibles », Revue du Crémis, 3(3), p. 32-40.

Bastien, R. et L. Turcotte (2010a). « Analyse en distance : bilan et prospective des interventions en milieu scolaire », Document de travail en diffusion restreinte.

Bastien, R. et L. Turcotte (2010b). «Étude exploratoire de l'approche École en santé sur le territoire de Montréal à partir du prisme d'agents pivots : première incursion dans le monde de cette pratique », Document de travail en diffusion restreinte.

Beck, U. (2003). La société du risque, Paris, Flammarion.

Bourdieu, P. (1993). La misère du monde, Paris, Seuil.

Castel, R. (1995). Les métamorphoses de la question sociale, Paris, Gallimard, coll. Folio Essais.

Castel, R. (2007). La discrimination négative : citoyens ou indigènes, Paris, La République des idées et Seuil.

Corbin, A. (2008). Le miasme et la jonquille, Paris, Flammarion, coll. Champs Histoire.

Coulon, A. (1993). Ethnométhodologie en éducation, Paris, PUF.

Donzelot, J. (2005). La police des familles, Éditions de Minuit.

Douglas, M. (1992). Risk and Blame: Essays in Cultural Theory, Londres, Routledge.

Faure, A. (1978). "Classe malpropre, classe dangereuse? Quelques remarques à propos des chiffonniers parisiens au XIX ${ }^{\mathrm{e}}$ siècle et de leurs cités », dans L. Murard et P. Zylberman (dir.), L'haleine des faubourgs : ville, habitat et santé au XIX siècle, revue Recherches (Paris), $\mathrm{n}^{\circ}$ 29, p. 79102.

Fleurbaey, M., N. Herpin, M. Martinez et D. Verger (1998). «Mesurer la pauvreté », Économie et statistique, 308(1), p. 23-33.

Foucault, M. (1975). Surveiller et punir : Naissance de la prison, Paris, NRF.

Gagnon, R. (1996). Histoire de la Commission des écoles catholiques de Montréal, Montréal, Boréal.

Guimond, S. (1994). "Les groupes sociaux», dans R. J. Vallerand (dir.), Les fondements de la psychologie sociale, Montréal, Gaëtan Morin, p. 655-705.

Miron, J.-F. (2004). « La difficile reconnaissance des parents », Recherche et formation, ${ }^{\circ}$ 47, p. 55-68.

Moles, O. C. (1993). «Collaboration between schools and disadvantaged parents: Obstacles and opening », dans N. F. Chavkin (dir.), Families and Schools in a Pluralistic Society, New York, State University of New York Press, p. 21-52. 
Moore, S. et S. Lasky (2001). «La participation des parents à l'éducation », Toronto, Office de la qualité et de la responsabilité en éducation (OQRE), Série d'études de recherche $n^{\circ} 6$.

Murard, L. et P. Zylberman (1978). « Hygienic preservative against various maladies », dans L. Murard et $P$. Zylberman (dir.), L'haleine des faubourgs : ville, habitat et santé au XIX $X^{e}$ siècle, revue Recherches (Paris), $n^{\circ} 29$, p. 7-22.

Murard, L. et P. Zylberman (1996). L'hygiène dans la République. La santé publique en France, ou l'utopie contrariée : 1870-1918, Paris, Fayard.

Paquin, J. (1960). Morale et médecine, Montréal, L'Immaculée-Conception et le Comité des Hôpitaux du Québec.

Perreault, M. (2008). " Une vulnérabilisation qui rend vulnérable au VIH/sida », dans V. Châtel et S. Roy (dir.), Penser la vulnérabilité: Visages de la fragilisation du social, Québec, Les Presses de l'Université du Québec, p. 149-163.

Pierrard, P. (2005). Les pauvres et leur histoire - de Jean Valjean à l'abbé Pierre, Paris, Bayard.

René, J.-F., I. Laurin et N. Dallaire (2009). « Faire émerger le savoir d'expérience de parents pauvres: forces et limites d'une recherche participative », Recherches qualitatives, 28(3), p. 40-63.

Warin, P. (2010). « Ciblage, stigmatisation et non-recours », Revue du Crémis, 3(2), p. 16-22. 